\title{
Preparation of a novel composite nanofiber gel-encapsulated human placental extract through layer-by-layer self-assembly
}

\author{
GUOHUI LIU, XI CHEN, WU ZHOU, SHUHUA YANG, SHUNAN YE, FAQI CAO, YI LIU and YUAN XIONG \\ Department of Orthopedics, Union Hospital, Tongi Medical College, \\ Huazhong University of Science and Technology, Wuhan, Hubei 430022, P.R. China
}

Received November 4, 2014; Accepted December 2, 2015

DOI: $10.3892 /$ etm.2016.3084

\begin{abstract}
Aqueous human placenta extract (HPE) has been previously used to treat chronic soft tissue ulcer; however, the optimal dosage of HPE has yet to be elucidated. The present study investigated a novel nanofiber gel composed through layer-by-layer (LbL) self-assembly, in which HPE was encapsulated. IKVAV, RGD, RAD16 and FGL-PA were screened and combined to produce an optimal vehicle nanofiber gel through LbL assembly. Subsequently, the aqueous HPE was encapsulated into this nanofiber at the appropriate concentration, and the morphology, particle size, drug loading efficacy, encapsulation rate, release efficiency and structure validation were detected. The encapsulation efficiency of all three HPE samples was $>90 \%$, the nanofiber gel exhibited a slow releasing profile, and the structure of HPE encapsulated in the nanofiber gel was unvaried. In conclusion, this type of novel composite nanocapsules may offer a promising delivery system for HPE.
\end{abstract}

\section{Introduction}

The wound healing potency of an aqueous human placental extract (HPE) has been clinically established in previous studies $(1,2)$. HPE has numerous applications in the treatment of chronic soft tissue ulcers and in the stimulation of endogenous growth factors, while it possesses anti-inflammatory and antimicrobial properties $(1,2)$. HPE efficiently activates adenosine $\mathrm{A} 2 \mathrm{~A}$ receptors and is not degraded in vivo, meaning it is more efficient than other types of therapy, including vacuum sealing drainage, local application of growth factor, and traditional Chinese medicine (3). However, the use of

Correspondence to: Dr Xi Chen or Dr Wu Zhou, Department of Orthopedics, Union Hospital, Tongi Medical College, Huazhong University of Science and Technology, 1277 Jiefang Avenue, Wuhan, Hubei 430022, P.R. China

E-mail: chenxichxi@126.com

E-mail: zhouwuzhw@126.com

Key words: novel layer-by-layer self-assembly, nanocomposite gel fiber, aqueous extract of human placenta appropriate vehicles to maintain the biological activity of HPE during the delivery process is crucial to the success of the treatment $(4,5)$.

Short poly-N-acetyl glucosamine (sNAG) nanofibers have been found to activate the integrin receptor on the surface of platelets, promote the formation of fibrous protein complexes and cell proliferation and migration, and stimulate the release of a variety of growth factors $(6,7)$. The activated integrin receptor was also found to increase the expression of vascular endothelial growth factor (VEGF) and accelerate the formation of new blood vessels through a transcriptional factor-dependent signaling pathway (8). Furthermore, sNAG nanofibers may also accelerate the expression of $\alpha$-defensins and defensin $\beta-1$ in endothelial and keratin cells (9). Therefore, self-assembled sNAG nanofiber gel encapsulating aqueous HPE can serve as an ideal matrix material and tool for the treatment of chronic soft tissue ulcers $(9,10)$.

Layer-by-layer (LbL) self-assembly is a widely used technique of alternating the adsorption of materials onto a surface using complementary interactions, one layer of material at a time, in order to create nanometer-thin films $(11,12)$. Due to its versatility and simplicity, the LbL self-assembly technique has been under intensive investigation for a wide range of purposes, including bone tissue engineering for vaccine delivery and the creation of biological interfaces $(13,14)$. The development of stimuli-responsive LbL-assembled nanoparticles has advanced in recent years (15).

In the present study, the aqueous HPE was encapsulated in a novel nanocomposite gel fiber $(16,17)$ prepared by the ideal formulation of IKVAV, RGD, RAD16 and FGL-PA through LbL self-assembly $(17,18,19,20)$, and the morphology, particle size, drug loading, drug release efficacy, encapsulation rate and structure validation were examined. IKVAV is an amphiphilic molecule, also known as isoleucine-lysine-valine-alanin-valine. The sequence of IKVAV is $\mathrm{C}_{16} \mathrm{H}_{31} \mathrm{O}-\mathrm{NH}-\mathrm{AAA}$ GGG E IKVAV-COOH, and this was prepared by the solid phase synthesis method (21). RAD16 is a self-assembled peptide composed of alternating negative and positive amino acids (ACN-RADARADARADARADA- $\mathrm{CONH}_{2}$ ). In addition, the FGL-PA sequence was $\mathrm{C}_{22} \mathrm{H}_{43} \mathrm{O}-\mathrm{NH}$-AAAGGGEVY VVAENQQGKSKA-COOH, and the RGD sequence was $\mathrm{C}_{16}$ $\mathrm{H}_{31} \mathrm{O}-\mathrm{NHAAAAGGGS}\left(\mathrm{PO}_{4}\right)$-RGD-COOH. The study aimed to investigate whether this novel type of composite nanocapsules may offer a promising delivery system for HPE. 


\section{Materials and methods}

Materials. IKVAV (relative molecular mass of 1,351.6 with 98\% purity; batch number, 20140520; Shanghai Bootech Bioscience \& Technology Co., Ltd., Shanghai, China), RAD16 (relative molecular mass of 1207.34 with $98 \%$ purity; Shanghai Bootech Bioscience \& Technology Co., Ltd.), FGL-PA (relative molecular mass of $2,485.92$ with $98 \%$ purity; Shanghai Bootech Bioscience \& Technology Co., Ltd.), and RGD (relative molecular mass of 1,207.34 with 99\% purity; Shanghai Bootech Bioscience \& Technology Co., Ltd.) were used in the present study.

Preparation of vehicle nanofiber gel. For the preparation of the vehicle nanofiber gel, $10 \mathrm{mg}$ IKVAV, $10 \mathrm{mg}$ RAD16, $10 \mathrm{mg}$ FGL-PA and $10 \mathrm{mg}$ RGD were added to a mixture of $0.1 \mathrm{M} \mathrm{NaOH}(400 \mu \mathrm{l})$ and twice-distilled water $(400 \mu \mathrm{l})$. The mixture was then placed in $37^{\circ} \mathrm{C}$ for $30 \mathrm{~min}$ and stirred vigorously until a clear liquid was obtained. The $\mathrm{pH}$ value was detected using a $\mathrm{pH}$ meter (pH 9.4) and $0.1 \mathrm{M} \mathrm{HCl}$ was used to adjust the $\mathrm{pH}$ to 8.5-9.0. Distilled water was used to adjust the peptide concentration to $1 \mathrm{mg} / \mathrm{ml}$. Next, $0.1 \mathrm{ml}$ Dulbecco's modified Eagle's medium/nutrient mixture F12 (DMEM-F12; Gibco; Thermo Fisher Scientific, Inc., Waltham, MA, USA) was added to $0.1 \mathrm{ml}$ peptide to trigger self-assembly.

A greater nanofiber gel viscosity resulted in better performance of the gel. Based on the preliminary results, IKVAV, FGL-PA and RGD were used for the formula screening (22). The appropriate proportion and concentration of IKVAV, FGL-PA and RGD were screened to prepare the optimum nanofibers gel for the appropriate times as follows: i) IKVAV:RGD = 2:1 (4.5 sec); ii) IKVAV:RGD = 1:1 (4 sec); iii) IKVAV:RGD = 1:2 (5 sec); iv) IKVAV:FGL-PA $=2: 1$ $(5.5 \mathrm{sec})$; v) IKVAV:FGL-PA = 1:1 (6 $\mathrm{sec})$; vi) IKVAV:FGL-PA = 1:2 (7 sec); vii) RGD:FGL-PA=2:1 $(8 \mathrm{sec})$; viii) RGD:FGL-PA = 1:1 (8.2 sec); ix) RGD:FGL-PA = 1:2 $(8.5 \mathrm{sec}) ; \quad \mathrm{x})$ IKVAV:RGD:FGL-PA $=1: 1: 1$ $(11 \mathrm{sec})$; xi) IKVAV:RGD:FGL-PA = 3:2:1 (9 sec); xii) IKVAV:RGD:FGL-PA = 1:2:3 (10 sec).

Preparation of nanofiber gel-encapsulated HPE. HPE was obtained from a 28 year-old patient recruited from the Union Hospital of the Tongi Medical College (Wuhan, China) on February 2014. According to the appropriate proportion and concentration, IKVAV, FGL-PA and RGD were added into the HPE, and then mixed with an equal volume DMEM-F12 to trigger self-assembly at room temperature. The solution was then diluted 0,50 and 100 times with distilled water and observed at magnifications of x30,000, x39,000, x65,000, $\mathrm{x} 93,000$ and $\mathrm{x} 135,000$ using a Tecnai-10 transmission electron microscope (Philips, Amsterdam, Netherlands).

Transmission electron microscopy. The morphology of the HPE nanofiber gels was analyzed using a Tecnai-10 transmission electron microscope (Philips). Briefly, following dilution (1:100) in water, the sample was negatively-stained with $1 \%$ (weight/volume) phosphotungstic acid (Shanghai Muhong Industrial Co., Ltd., Shanghai, China) for $5 \mathrm{~min}$ at $25^{\circ} \mathrm{C}$. Subsequently, the sample was placed on copper film grids
(Wuhan Boster Biological Technology, Ltd., Wuhan, China) and observed using transmission electron microscopy after drying for $10 \mathrm{~min}$ at $25^{\circ} \mathrm{C} \mathrm{(23).}$

Particle size determination. After diluting 100-fold with distilled water, the mean particle size of the HPE nanofiber gel was determined using Zetasizer 3000 HS (Malvern Instruments Ltd., Malvern, UK). Three parallel measurements were performed for each sample as previously described (24).

Encapsulation efficiency and drug loading testing of HPE nanofiber gel. The encapsulation efficiency and drug loading was analyzed by dissolving $~ 5 \mathrm{mg}$ HPE nanofiber gel in either $1.2 \mathrm{ml}$ distilled water or absolute ethyl alcohol. Next, the mixture was centrifuged at $8,000 \mathrm{x}$ g for $10 \mathrm{~min}$ and the precipitation was resuspended in $1.2 \mathrm{ml}$ distilled water. The supernatant and precipitation were detected using an ultraviolet (UV) detector (Alpha 1500; Shanghai Lab-Spectrum Instruments Co., Ltd., Shanghai, China) at $280 \mathrm{~nm}$. The formula used to measure the encapsulation efficiency was as follows: Encapsulation efficiency $=$ (concentration of supernatant + concentration of precipitation in demulsified samples - concentration of precipitation in non-demulsified samples)/total concentration of demulsified samples. The formula used to measure drug loading was as follows: Drug loading $=$ actual drug concentration / (drug-loading particle + drug concentration). Three parallel measurements were performed for each sample.

Slow-releasing potential detection of HPE nanofiber gel. The slow-releasing potential of the HPE nanofiber gel was evaluated using a dialysis method. Briefly, dialysis bags (molecular weight cut-off, 1,000 Da) containing $0.1 \mathrm{ml}$ HPE nanofiber gel were immersed in a thermostatic gas bath containing $20 \mathrm{ml}$ release medium (phosphate-buffered saline, $\mathrm{pH} 7.2$ ) at $37^{\circ} \mathrm{C}$. At predefined intervals $(0.25,0.5,1,1.5,2,4,6,9,12,24$, 48, 72, and $96 \mathrm{~h}$ ), $1 \mathrm{ml}$ aliquots of phosphate-buffered saline (Wuhan Boster Biological Technology, Ltd., Wuhan, China) were withdrawn and replaced with the same volume of release medium. The HPE release behavior of the nanofiber gel was determined using a UV spectrophotometer (Agilent 8453; Agilent Technologies, Santa Barbara, CA, USA) at $280 \mathrm{~nm}$. The measured concentrations were calculated according to the standard curve, followed by calculation of the cumulative release rate (22).

Crystal structure validation of HPE nanofiber gel. The HPE stock solution and nanofiber gel were diluted 100 times with distilled water. The solution was stirred for $10 \mathrm{~min}$ at $20 \mathrm{x} \mathrm{g}$. The crystal structure of the HPE stock solution and HPE nanofiber gel was then evaluated using an X-ray diffractometer (Siemens D5000; Siemens AG, Munich, Germany). The condition of the diffraction was as follows: $\lambda=1.5064 \mathrm{~A}^{\circ}$; voltage, $20 \mathrm{kV}$; electric current, $20 \mathrm{~mA} ; 2 \theta$ scanning in the range of $10^{\circ}$ to $80^{\circ}$; scanning frequency, $0.02^{\circ} \mathrm{Q} / \mathrm{S}$.

Statistical analysis. Statistical analysis was performed using the SPSS version 16 software (SPSS, Inc., Chicago, IL, USA). The statistical significance of differences was determined 


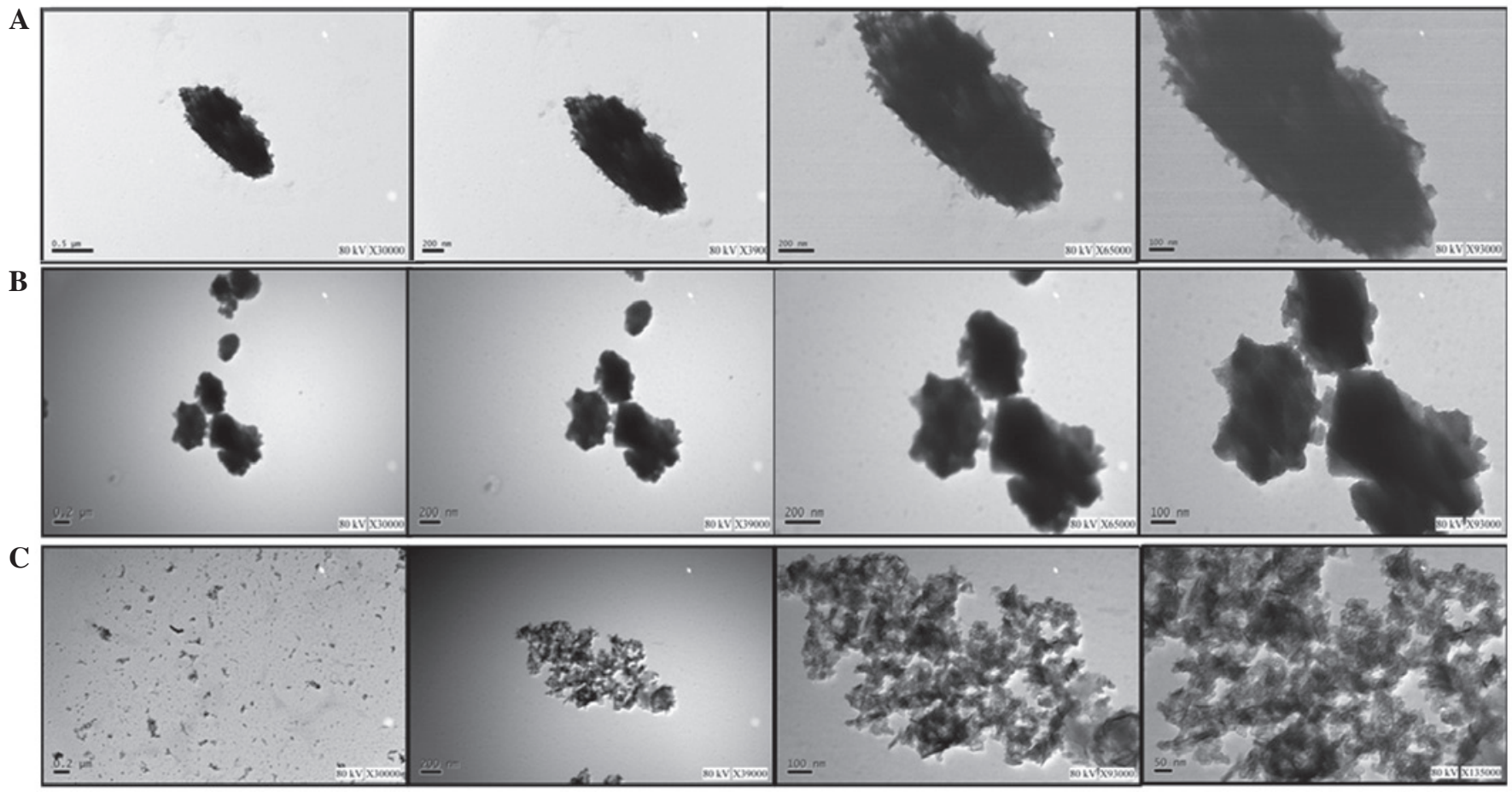

Figure 1. Morphology of human placental extract encapsulated in nanofibers, as observed using transmission electron microscopy. Different dilutions were used, including dilution by (A) 0, (B) 50 and (C) 100 times. The four images in each part of the figure were captured at different magnifications. In (A) and (B), the magnification is $\times 30,000, x 39,000, x 65,000$ and $\times 93,000$ (from left to right), while in (C) the magnifications are x30,000, x39,000, x93,000 and x135,000 (from left to right).

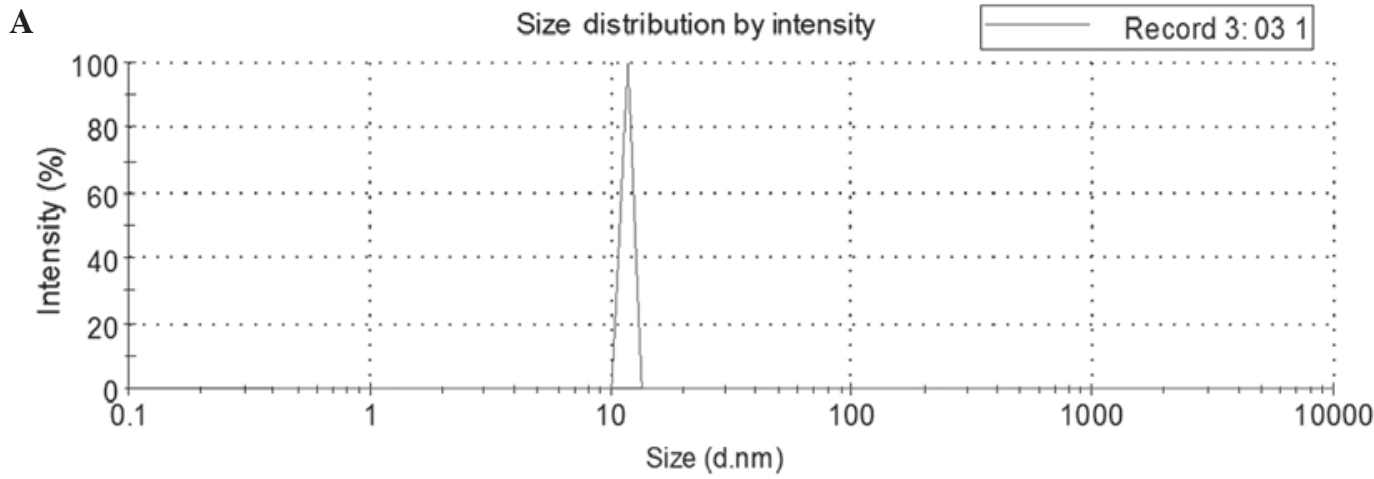

B

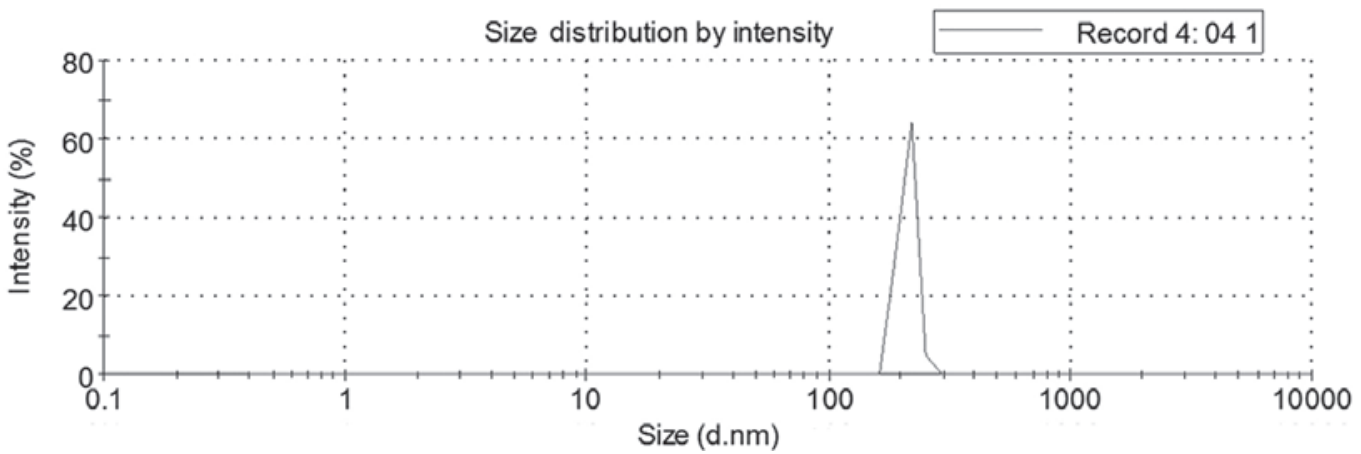

Figure 2. Mean particle size of (A) vehicle nanofiber gel and (B) human placental extract nanofiber gel.

using one-way analysis of variance. All statistical tests were two-sided, and $\mathrm{P}<0.05$ was considered to indicate a statistically significant difference. Results are presented as the mean \pm standard deviation.

\section{Results}

Preparation and characterization of HPE encapsulated in nanofiber gels. The IKVAV, RAD16, FGL-PA and RGD 


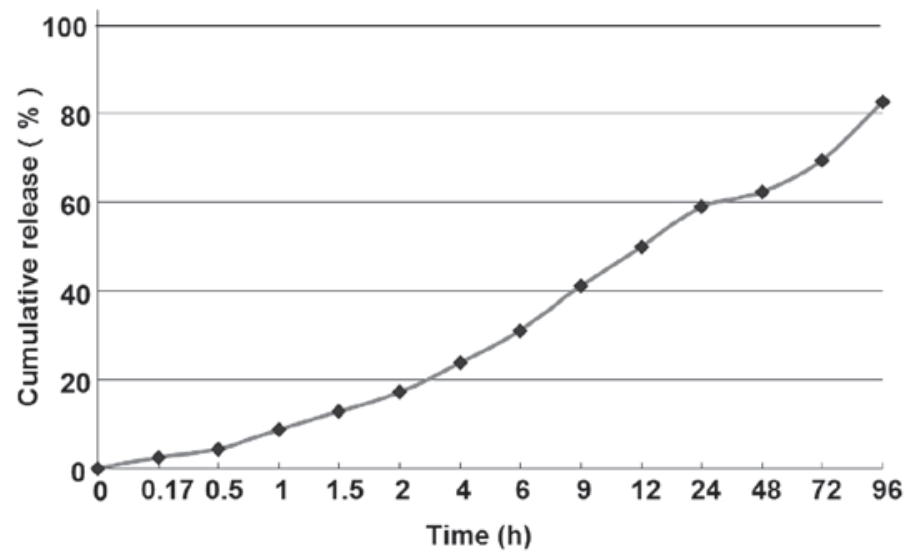

Figure 3. In vitro release profiles of the HPE encapsulated in nanofiber gels in phosphate-buffered saline (pH 7.2) at $37^{\circ} \mathrm{C}$.

peptides were synthesized and used to form nanofiber skeletons using the self-assembly method via electrostatic and hydrophobic interactions. After a preliminary screening, IKVAV, RGD and FGL-PA were able to form nanofiber skeletons with greater viscosity. Twelve different combinations of IKVAV, RGD and FGL-PA were used for the formula screening. The results indicated that the optimal proportion of the nanofiber gel was IKVAV : RGD : FGL-PA = 1:1:1 and the optimal concentration was $0.6 \mathrm{mg} / \mathrm{ml}$, as determined by observing the liquidity, diffusion time and viscosity.

Subsequently, the HPE was encapsulated into the nanofibers. The morphology of the HPE nanofibers was observed using transmission electron microscopy. The results revealed that the HPE nanofibers had a fibrous appearance under different dilutions. With the increase of the dilution, the nanofiber gel appeared to be more homogeneously dispersed. At a dilution of 100, the nanofiber gel was found to be directly observed (Fig. 1).

As shown in Fig. 2, the mean particle widths of the vehicle and of the HPE nanofiber gels were $\sim 11.7$ and $212 \mathrm{~nm}$, respectively.

Encapsulation efficiency and in vitro release of HPE. The results of UV spectrophotometric analysis revealed that the encapsulation efficiency of all three HPE samples (dilution performed in triplicate) was $>90 \%$ (96.8, 98.3 and 94.8\%), with a mean encapsulation efficiency of $96.6 \pm 1.75 \%$. The drug loading of the three samples (dilution performed in triplicate) was found to be $5.2,5.13$ and $5.11 \mathrm{mg} / \mathrm{g}$, with a mean drug loading of $5.15 \pm 0.04 \mathrm{mg} / \mathrm{g}$ (data not shown).

The in vitro release of HPE nanofiber gel was examined using a dialysis method. The drug release profile was performed under simulated physiologic conditions ( $\mathrm{pH}$ 7.2). As shown in Fig. 3, the HPE nanofiber gel showed a sustained drug release profile, with a total HPE release from the nanofibers of $2.35-82.58 \%$. The nanofiber gel exhibited a slow-releasing profile ( $>4$ days).

Crystal structure validation of HPE nanofiber gel. The results indicated that the peak number and time of HPE nanofiber gel was similar to that of the HPE stock solution. The preliminary results confirmed that the structure of HPE encapsulated in the nanofiber gel was unvaried compared with the HPE structure.

\section{Discussion}

The absolute or relative reduction of exogenous growth factor and its receptor is one of the main reasons why the treatment of chronic soft tissue ulcer is challenging $(25,26)$. The topical application of active growth factors can promote the healing of chronic ulcer (27); however, our previous study revealed that the exogenous growth factors were easily degraded and diluted due to their short half-life (28). Signaling pathways that are able to awaken the body's self-healing mechanisms are therefore required to promote the sustained release of endogenous growth factors. Recent studies have shown that adenosine A2A receptor activation may play an important role in the protection and promotion of wound healing in ischemia, hypoxia, inflammation, trauma and numerous other pathological processes $(29,30)$. The adenosine A2A receptor activation can inhibit the generation of reactive oxygen species and promote the release of cytokines, such as tumor necrosis factor- $\alpha$, accelerate osteoblast, fibroblast and fat precursor cell proliferation, as well as the release of VEGF, angiogenin and glutamine transferase II, and thus promoting wound healing $(31,32)$. Therefore, selective activation of adenosine A2A receptors awakens the body's self-healing mechanisms (33).

Numerous studies have demonstrated the biological actions of HPE in various diseases, and HPE has been used in wound healing in several countries for years as a folk remedy $(34,35)$. Another study suggested that HPE exerts an anti-inflammatory function that suppresses chemical mediators, and that these effects may be associated with innate immune functions (36). Furthermore, previous studies indicated that oligomeric DNA nucleotides, such as polydeoxyribonucleotide (PDRN), are linear DNA nucleotide polymers from HPE with a length range of 50-2,000 bp $(37,38)$. PDRN can efficiently activate adenosine A2A receptors, without being degraded into small fragments, thus preventing the activation of other adenosine receptors $(39,40)$. PRDN treatment for chronic soft tissue ulcer has been found to significantly increase wound granulation, thus promoting wound healing (41). However, the method used to promote the PDRN late release will directly affect its therapeutic effect on the chronic soft tissue ulcer.

To date, limited information is available regarding the dosage of HPE required for wound healing. In the present study, aqueous HPE was encapsulated in a novel nanofiber 
gel prepared by the appropriate formulation of IKVAV, RGD, RAD16 and FGL-PA through LbL assembly. Subsequently, the morphology, particle size, drug loading efficacy, encapsulation rate, release efficacy and structure validation were examined. The results showed that the particle size of the HPE nanofiber gel was $212 \mathrm{~nm}$, which was included in the 1-1,000 nanometer materials. Following the detection of the encapsulation efficiency and drug loading, the HPE nanofiber gel was found to have high drug loading $(5.15 \mathrm{mg} / \mathrm{g})$ and high encapsulation efficiency (96.6\%). Furthermore, the HPE nanofiber gel exhibited a slow-releasing profile ( $>4$ days). Investigation of the crystal diffraction structure confirmed that the structure of HPE that was encapsulated in the nanofiber gel did not undergo any apparent changes. Therefore, these novel composite nanocapsules may offer a promising delivery system for HPE.

\section{Acknowledgements}

The present study was supported by the National Natural Science Fund of China (grant no. 81271968).

\section{References}

1. De D, Chakraborty PD and Bhattacharyya D: Regulation of trypsin activity by peptide fraction of an aqueous extract of human placenta used as wound healer. J Cell Physiol 226: 2033-2040, 2011.

2. De D, Chakraborty PD and Bhattacharyya D: Analysis of free and bound NADPH in aqueous extract of human placenta used as wound healer. J Chromatogr B Analyt Technol Biomed Life Sci 877: 2435-2442, 2009.

3. Xu Li-jie, Wang Zhi-ping. An analysis for 50 examples of curative effect about ulcer powder for external use curing skin soft tissue injury. China Practical Medicine 3: 13-14, 2008.

4. Nath S and Bhattacharyya D: Cell adhesion by aqueous extract of human placenta used as wound healer. Indian J Exp Biol 45: 732-738, 2007.

5. Chakraborty PD and Bhattacharyya D: Isolation of fibronectin type III like peptide from human placental extract used as wound healer. J Chromatogr B Analyt Technol Biomed Life Sci 818: 67-73, 2005.

6. Beeson CC, Beeson GC, Buff H, Eldridge J, Zhang A, Seth A, Demcheva M, Vournakis JN and Muise-Helmericks RC: Integrin-dependent Akt1 activation regulates PGC-1 expression and fatty acid oxidation. J Vasc Res 49: 89-100, 2012.

7. Muise-Helmericks RC, Demcheva M, Vournakis JN and Seth A: Poly-N-acetyl glucosamine fibers activate bone regeneration in a rabbit femur injury model. J Trauma 71 (2 Suppl 1): S194-S196, 2011.

8. Erba P, Adini A, Demcheva M, Valeri CR and Orgill DP: Poly-N-acetyl glucosamine fibers are synergistic with vacuum-assisted closure in augmenting the healing response of diabetic mice. J Trauma 71 (2 Suppl 1) S187-S193, 2011.

9. Lindner HB, Zhang A, Eldridge J, Demcheva M, Tsichlis P, Seth A, Vournakis J and Muise-Helmericks RC: Anti-bacterial effects of poly-N-acetyl-glucosamine nanofibers in cutaneous wound healing: Requirement for Akt1. PloS One 6: e18996, 2011.

10. Scherer SS, Pietramaggiori G, Matthews J, Perry S, Assmann A, Carothers A, Demcheva M, Muise-Helmericks RC, Seth A Vournakis JN, et al: Poly-N-acetyl glucosamine nanofibers: A new bioactive material to enhance diabetic wound healing by cell migration and angiogenesis. Ann Surg 250: 322-330, 2009.

11. Jiang F, Yeh CK, Wen J and Sun Y: N-trimethylchitosan/alginate layer-by-layer self-assembly coatings act as 'fungal repellents' to prevent biofilm formation on healthcare materials. Adv Healthc Mater 4: 469-475, 2015.

12. Lv H, Chen Z, Yang X, Cen L, Zhang X and Gao P: Layer-by-layer self-assembly of minocycline-loaded chitosan/alginate multilayer on titanium substrates to inhibit biofilm formation. J Dent 42: 1464-1472, 2014.
13. Lee H, Hong D, Choi JY, Kim JY, Lee SH, Kim HM, Yang SH and Choi IS: Layer-by-layer-based silica encapsulation of individual yeast with thickness control. Chem Asian J 10: 129-132, 2015.

14. Choi Y, Choi S, Jeong HY, Liu M, Kim BS and Kim G: Highly efficient layer-by-layer-assisted infiltration for high-performance and cost-effective fabrication of nanoelectrodes. ACS Appl Mater Interfaces 6: 17352-17357, 2014.

15. Huang Z, Yao Y, Han L and Che S: Control of chiral nanostructures by self-assembly of designed amphiphilic peptides and silica biomineralization. Chemistry 20: 17068-17076, 2014.

16. Shah RN, Shah NA, Del Rosario Lim MM, Hsieh C, Nuber G and Stupp SI: Supramolecular design of self-assembling nanofibers for cartilage regeneration. Proc Natl Acad Sci USA 107: 3293-3298, 2010

17. Sun X, Xu Y, Wang C and Li Q: Self-assembly of RGD peptide nanofiber hydrogels and its biocompatibility studies combined with adipose derived stem cells. Acta Universitatis Medicinalis Anhui 46: 1253-1256, 2011.

18. Sehgal RR and Banerjee R: IKVAV-functionalized self-assembling peptide hydrogel for improved neural stem cell transplantation. Nanomedicine (Lond) 8: 521-522, 2013.

19. Zhang Z, Zheng Q, Wu Y and Wu B. Biocompatibility of FGL peptide self-assembly nano-fibers with neural stem cells in vitro. Chinese Journal of Reconstructive surgery 22: 1369-1372, 2008 (In Chinese).

20. Martínez-Ramos C, Arnal-Pastor M, Vallés-Lluch A and Pradas MM. Peptide gel in a scaffold as a composite matrix for endothelial cells. J Biomed Mater Res A 103: 3293-3302, 2015.

21. Yao C, Hedrick M, Pareek G, Renzulli J, Haleblian G and Webster TJ: Nanostructured polyurethane-poly-lactic-co-g lycolic acid scaffolds increase bladder tissue regeneration: An in vivo study. Int J Nanomedicine 8: 3285-3296, 2013.

22. Gu X, Wang J, Wang Y, Wang Y, Gao H and Wu G: Layer-by-layer assembled polyaspartamide nanocapsules for $\mathrm{pH}$-responsive protein delivery. Colloids Surf B Biointerfaces 108: 205-211, 2013.

23. Parwe SP, Chaudhari PN, Mohite KK, Selukar BS, Nande SS and Garnaik B: Synthesis of ciprofloxacin-conjugated poly (L-lactic acid) polymer for nanofiber fabrication and antibacterial evaluation. Int J Nanomedicine 9: 1463-1477, 2014.

24. Qi R, Guo R, Zheng F, Liu H, Yu J and Shi X: Controlled release and antibacterial activity of antibiotic-loaded electrospun halloysite/poly (lactic-co-glycolic acid) composite nanofibers. Colloids Surf B Biointerfaces 110: 148-155, 2013.

25. Coull AF, Atherton I, Taylor A and Watterson AE: Prevalence of skin problems and leg ulceration in a sample of young injecting drug users. Harm Reduct J 11: 22, 2014.

26. Okrainec K, Booth GL, Hollands S and Bell CM: Impact of language barriers on complications and mortality among immigrants with diabetes: A population-based cohort study. Diabetes Care 38: 189-196, 2015.

27. Levy A, Kopplin K and Gefen A: An air-cell-based cushion for pressure ulcer protection remarkably reduces tissue stresses in the seated buttocks with respect to foams: Finite element studies. J Tissue Viability 23: 13-23, 2014

28. Liu G, Wang J, Yang S, Xu W, Ye S and Xia T: Effect of a porous tantalum rod on early and intermediate stages of necrosis of the femoral head. Biomed Mater 5: 065003, 2010.

29. Keränen H, Gutiérrez-de-Terán H and Aqvist J: Structural and energetic effects of A2A adenosine receptor mutations on agonist and antagonist binding. PloS One 9: e108492, 2014.

30. Kim DG and Bynoe MS: A2A adenosine receptor regulates the human blood-brain barrier permeability. Mol Neurobiol 52: 664-678, 2015.

31. Metsola J, Maksimow M, Ojaniemi M, Metsola $H$, Marttila-Ichihara F, Vuolteenaho R, Yegutkin GG, Salmi M, Hallman M and Jalkanen S: Postnatal development and LPS responsiveness of pulmonary adenosine receptor expression and of adenosine-metabolizing enzymes in mice. Pediatr Res 76: 515-521, 2014.

32. Beggiato S, Antonelli T, Tomasini MC, Borelli AC, Agnati LF, Tanganelli S, Fuxe K and Ferraro L: Adenosine A2A-D2 receptor-receptor interactions in putative heteromers in the regulation of the striato-pallidal GABA pathway: Possible relevance for Parkinson's disease and its treatment. Curr Protein Pept Sci 15: 673-680, 2014.

33. Fuxe K, Borroto-Escuela D, Fisone G, Agnati LF and Tanganelli S: Understanding the role of heteroreceptor complexes in the central nervous system. Curr Protein Pept Sci 15: 647, 2014. 
34. Mitsui Y, Bagchi M, Marone PA, Moriyama H and Bagchi D: Safety and toxicological evaluation of a novel, fermented, peptide-enriched, hydrolyzed swine placenta extract powder. Toxicol Mech Methods 25: 13-20, 2015.

35. Bogacz B, Bartkowiak-Wieczrek J, Mikołajczak PŁ, Rakowska-Mrozikiewicz B, Grześkowiak E, Wolski H, Czerny B and Mrozikiewicz PM: The influence of soybean extract on the expression level of selected drug transporters, transcription factors and cytochrome $\mathrm{P} 450$ genes encoding phase I drug-metabolizing enzymes. Ginekol Pol 85: 348-353, 2014.

36. Mrozikiewicz PM, Bogacz A, Czerny B, Karasiewicz M, Kujawski R, Mikolajczak PL, Seremak-Mrozikiewicz A, Grzeskowiak E and Bobkiewicz-Kozlowska T: The influence of a standardized soybean extract (Glycine max) on the expression level of cytochrome P450 genes in vivo. Ginekol Pol 81: 516-520, 2010.

37. Bitto A, Polito F, Irrera N, D'Ascola A, Avenoso A, Nastasi G, Campo GM, Micali A, Bagnato G, Minutoli L, Marini H, et al: Polydeoxyribonucleotide reduces cytokine production and the severity of collagen-induced arthritis by stimulation of adenosine $\mathrm{A}\left({ }_{2} \mathrm{~A}\right)$ receptor. Arthritis Rheum 63: 3364-3371, 2011.
38. Minutoli L, Arena S, Bonvissuto G, Bitto A, Polito F, Irrera N, Arena F, Fragalà E, Romeo C, Nicotina PA, et al: Activation of adenosine A2A receptors by polydeoxyribonucleotide increases vascular endothelial growth factor and protects against testicular damage induced by experimental varicocele in rats. Fertil Steril 95: 1510-1513, 2011.

39. Squadrito F, Bitto A, Altavilla D, Arcoraci V, De Caridi G, De Feo ME, Corrao S, Pallio G, Sterrantino C, Minutoli L, et al: The effect of PDRN, an adenosine receptor A2A agonist, on the healing of chronic diabetic foot ulcers: Results of a clinical trial. J Clin Endocrinol Metab 99: E746-E753, 2014.

40. Bitto A, Oteri G, Pisano M, Polito F, Irrera N, Minutoli L, Squadrito $F$ and Altavilla D: Adenosine receptor stimulation by polynucleotides (PDRN) reduces inflammation in experimental periodontitis. J Clin Periodontol 40: 26-32, 2013.

41. Minutoli L, Antonuccio P, Squadrito F, Bitto A, Nicotina PA, Fazzari C, Polito F, Marini H, Bonvissuto G, Arena S, Morgia G, et al: Effects of polydeoxyribonucleotide on the histological damage and the altered spermatogenesis induced by testicular ischaemia and reperfusion in rats. Int J Androl 35: 133-144, 2012. 